\title{
Recombinant OspC from Borrelia burgdorferi sensu stricto, $B$. afzelii and $B$. garinii in the serodiagnosis of Lyme borreliosis
}

\author{
JAANA PANELIUS*, PEKKA LAHDENNE $\dagger$, TERO HEIKKILÄ*†, MIIKKA PELTOMAA \\ JARMO OKSI§ and ILKKA SEPPÄLÄ*\|
}

\begin{abstract}
* Haartman Institute, Department of Bacteriology and Immunology, †Hospital for Children and Adolescents, $\$$ Department of Otorhinolaryngology, University of Helsinki, §Department of Medicine, Turku University Central Hospital, Turku and ||HUCH Laboratory Diagnostics, Helsinki University Central Hospital, Helsinki, Finland
\end{abstract}

\begin{abstract}
Genes for the outer-surface protein C (OspC) from three north European human isolates of Borrelia burgdorferi sensu stricto, $B$. afzelii and $B$. garinii were cloned and sequenced. Polyhistidine-tagged recombinant OspC (rOspC) proteins were produced in Escherichia coli and used, after biotinylation, as antigens on streptavidin-coated plates in enzyme-linked immunosorbent assays (ELISA). In IgM ELISA, 30\% (5/17) and 35\% (6/17) of patients with erythema migrans (EM) in the acute or convalescent phase, respectively, reacted with one to three rOspCs. Of the patients, $53 \%(8 / 15)$ with neuroborreliosis (NB) and 53\% (8/15) with Lyme arthritis (LA) had IgM antibodies to OspC. The immunoreactivity was stronger against $\mathrm{rOspC}$ from $B$. afzelii and $B$. garinii than against rOspC from B. burgdorferi sensu stricto. In early Lyme borreliosis (LB), rOspC and flagella performed equally well in detecting IgM antibodies. Cross-reactive antibodies to rOspC were observed in serum samples from patients with rheumatoid factor positivity and with syphilis or Epstein-Barr virus (EBV) infection. In IgM ELISA, thiocyanate in the serum dilution buffer reduced EBV-associated non-specific positive reactions. Of the patient sera examined in IgG ELISA, 30\% (5/17) with EM in the acute phase, 35\% (6/17) with EM in the convalescent phase, 33\% (5/15) with NB and $60 \%(9 / 15)$ with $\mathrm{LA}$ were positive. Because of the heterogeneity of OspC, a polyvalent antigen with several OspC variants from at least $B$. afzelii and $B$. garinii is needed to improve the sensitivity of OspC ELISA in the serodiagnosis of LB in Europe.
\end{abstract}

\section{Introduction}

Serodiagnostic tests for Lyme borreliosis (LB) are based mainly on enzyme-linked immunosorbent assays (ELISA), the antigens being borrelial whole-cell lysate (WCL) or flagella. Currently, because of insufficient specificity and sensitivity, these tests perform suboptimally and Western blot (WB) analyses on ELISA positive samples are needed to confirm the diagnosis [1]. Some viral infections cause false-positive results in IgM serology [2] and, during the early stages of LB, antibody responses are often absent or delayed [3]. Use of recombinantly produced borrelial antigens has increased the specificity of serological assays, but

Received 22 March 2002; accepted 4 April 2002.

Corresponding author: $\operatorname{Dr}$ J. Panelius (e-mail: jaana. panelius@helsinki.fi). sensitivity to single antigens has so far remained insufficient. Furthermore, new assays for serodiagnosis of LB are needed to discriminate LB from vaccination.

The outer-surface protein C (OspC) of Borrelia burgdorferi has been found to induce an early IgM response [4-9]. In people vaccinated with OspA, OspC antibody assay has been proposed for discrimination between infection with $B$. burgdorferi sensu stricto and a vaccination response [9]. In the serodiagnosis of European LB with WCL immunoblots, specific antibody responses to OspC of the three borrelial genospecies as part of a combination of antigens have been reported to increase sensitivity [10]. The best combination of antigens for IgM WCL immunoblot included OspC from B. afzelii and B. garinii strains. However, false positive $\operatorname{IgM}$ reactions to OspC have occurred both in ELISA [6,9] and in immunoblot [11] 
studies on patients with mononucleosis. In an IgM ELISA, Rauer et al. [8] reported improved specificity over WCL when recombinant OspC (rOspC) was combined with a $14-\mathrm{kDa}$ flagellin fragment. Two peptide antigens from the conserved regions of the OspC sequence have been evaluated in IgM ELISA, an amino-terminal peptide at position 9-22 [12] and a carboxy-terminal decapeptide [13]. Both peptide antigens have shown improved discrimination between LB patients and controls in preliminary studies.

In IgG serology, a single rOspC antigen has proved to be beneficial in some studies $[4,5,13,14]$ but has shown low sensitivity in others $[6,9]$. Recombinant chimeric borrelial proteins, including fragments from OspC, have also been studied as antigens in a combined IgG-IgM ELISA [15]. Recently, a rapid immunochromatographic assay based on these chimeric proteins was suggested to be as sensitive as, and more specific than, the commercial WCL ELISA [16].

A problem concerning the use of OspC as a diagnostic antigen is the extensive structural variation of this molecule (sequence identity ranging from $62 \%$ to $80 \%$ ) within, as well as between, subspecies of B. burgdorferi sensu lato [17-19]. European and North American isolates of B. burgdorferi sensu lato have been classified into at least 16 serotypes of OspC [20,21]: six OspC serotypes for B. burgdorferi sensu stricto, four for $B$. afzelii and six for $B$. garinii. In comparison, only one OspA serotype has been reported for each subspecies of B. burgdorferi sensu stricto and $B$. afzelii, and five for B. garinii [22].

The purpose of the present study was to evaluate the advantage of combining variant rOspC antigens from all three pathogenic borrelial species in an ELISA panel. This report describes the cloning and expression of OspC proteins from three European borrelial strains representing B. burgdorferi sensu stricto, B. afzelii and $B$. garinii, and the results of ELISA assays with these OspC recombinants as antigens. It also shows the reduction of non-specific reactions in rOspC IgM ELISA with sample buffer containing sodium thiocyanate $(\mathrm{NaSCN})$.

\section{Materials and methods}

\section{Borrelial strains}

The study used domestic borrelial strains of $B$. burgdorferi sensu stricto (ia) isolated from cerebrospinal fluid of a Finnish patient with neuroborreliosis, and of B. afzelii (A91) and B. garinii (40) isolated from skin biopsies of Finnish patients with erythema migrans. These strains were genotyped by PCR of the $f l a B$ and subsequent sequencing of the PCR product, as described previously [23].

\section{Borrelia culture and DNA isolation}

Borrelial strains were cultured in Barbour-StoennerKelly-H (BSK-H) medium (Sigma) at $33^{\circ} \mathrm{C}$ with a $\mathrm{CO}_{2}$ $5 \%$ atmosphere until growth was c. $(1-2) \times 10^{8}$ cells $/ \mathrm{ml}$. The genomic DNA was then isolated with the DNeasy Tissue Kit (Qiagen, Hilden, Germany).

\section{PCR and cloning of the genes}

For each borrelial strain, the $o s p C$ coding sequence was PCR-amplified from the genomic DNA (Table 1). Approximately $1 \mathrm{ng}$ of template DNA was used and the parameters in the PCR amplification reaction were 30 cycles at $94^{\circ} \mathrm{C}$ for $1 \mathrm{~min}, 50^{\circ} \mathrm{C}$ for $1 \mathrm{~min}$ and $72^{\circ} \mathrm{C}$ for 1.5 min with AmpliTaq Gold DNA Polymerase (Perkin Elmer, USA), followed by a final extension of $10 \mathrm{~min}$ at $72^{\circ} \mathrm{C}$. DNA products were visualised by gel electrophoresis on agarose 1\% NA gel (Amersham Pharmacia, Uppsala, Sweden) containing ethidium bromide. The PCR products were cloned into the pCR 2.1-TOPO vector (Invitrogen, Groningen, The Netherlands). The Escherichia coli host cell used for cloning was INF $\alpha \mathrm{F}$ (Invitrogen).

Table 1. Primers used in PCR reactions for $\operatorname{osp} C$ sequencing and expression of the respective protein

\begin{tabular}{llc}
\hline Target DNA & Primer $\left(5^{\prime}-3^{\prime}\right)$ & Location (bp) \\
\hline Sequencing & & \\
$1)$ & AAA AGG AGG CAC AAA TTA ATG & $-18-3$ \\
$2)$ & TAA AGC TAT TGG TAA AAA AAT A & $225-246$ \\
$3)$ & GTT GTG GCA GAA AGT CC & $604-620$ \\
$4)$ & TTG TAA GCT CTT TAA CTG AAT & $2269-2253$ \\
$5)$ & ATT GGC GCA TTA GTT AC & $2417-2399$ \\
$6)$ & GAA TCA ATC CAA AGA AAC A & \\
Expression & CCG GAT CCA ATA ATT CAG GGA AAG ATG G & $58-77$ \\
OspC Bbia & CC GTA CCG CCA AGA AAT CTT TCT TGA C & $718-698$ \\
& CCG GAT CCA ATA ATT CAG GGA AAG GTG G & $58-77$ \\
OspC $_{\text {BaA91 }}$ & CC G GA CCG CCA AGA AAT CTT TCT TGA C & $718-698$ \\
OspC $_{\text {Bg40 }}$ & CCG GAT CCA ATA ATT CAG GTG GGG ATA C & $58-77$ \\
& CCG GTA CCG CCA AGA AAT CTT TCT TGA C & $718-698$ \\
\hline
\end{tabular}

Underlining indicates Bam HI and KpnI cleavage sites. 


\section{DNA sequencing}

Plasmid DNA containing ospC inserts was isolated from E. coli with a QIAprep-spin plasmid kit (Qiagen). DNA sequencing with a DyePrimer (T7, M13Rev) cycle sequencing kit (Applied Biosystems, USA) was performed in accordance with the manufacturer's instructions by the Core Facility of the Haartman Institute, University of Helsinki. Sequencing reactions were run and analysed with an automated sequencing apparatus model 373A (Applied Biosystems). DNA and protein sequences were analysed with Lasergene software (DNASTAR, USA). To eliminate any errors caused by Taq polymerase, cloning and sequencing of the $\operatorname{osp} C$ genes were done twice.

\section{Construction of the expression plasmid and expression of $\mathrm{rOspC}$}

The $\operatorname{ss} C$ constructs comprised the coding sequence of the mature protein. The PCR products were cloned into the pCR 2.1-TOPO vector. After digestion of the purified pCR 2.1-TOPO plasmid with Bam HI and $K p n$ I, the cleaved $o s p C$ was ligated with T4 ligase into the similarly cut pQE-30 expression plasmid (Qiagen) that adds an N-terminal 6-histidine tag to the protein. The ligation mixture was used to transform $E$. coli M15 host cells, as described in the manufacturer's instructions (Qiagen). The transformation mixture was plated on to Luria-Bertani agar containing ampicillin 100 $\mu \mathrm{g} / \mathrm{ml}$ and kanamycin $25 \mu \mathrm{g} / \mathrm{ml}$. A primary culture for expression of the rOspC was started by inoculating $50 \mathrm{ml}$ of Luria-Bertani broth containing antibiotics as above with a single colony from a fresh transformant plate. The culture was incubated overnight at $37^{\circ} \mathrm{C}$ with shaking. This starter culture was diluted 1 in 100 to $1500 \mathrm{ml}$ with Luria-Bertani broth containing antibiotics as above and incubated at $37^{\circ} \mathrm{C}$ for $3 \mathrm{~h}$ (growth reached the mid-log phase; the $\mathrm{OD}_{600}$ was $c$. 0.6). Isopropyl- $\beta$ D-thiogalactoside (Calbiochem, USA) was added to a final concentration of $0.6 \mathrm{mM}$, and the culture was incubated for a further $3 \mathrm{~h}$. The cells were then harvested, washed and sonicated. The expressed rOspC was attached via its 6-histidine tag to a Chelating Sepharose Fast Flow column (Amersham Pharmacia) containing $\mathrm{Ni}^{2+}$. rOspC was subsequently eluted from the column with $0.16 \mathrm{M}$ imidazole eluting buffer. Protein expression and purity were confirmed by SDS-PAGE.

\section{Purification of rOspC and measurement of $\mathrm{rOspC}$ concentration}

rOspCs originating from strains ia, A91 or 40 (referred to as $\mathrm{rOspC}_{\mathrm{Bbia}}, \mathrm{rOspC}_{\mathrm{BaA} 91}$ or $\mathrm{rOspC}_{\mathrm{Bg} 40}$ ) were dialysed in $10 \mathrm{mM}$ diaminopropane- $\mathrm{HCl}, \mathrm{pH} \mathrm{8.8,} \mathrm{before}$ purification by chromatography, in which protein was loaded on to a Poros HQ 20 column (PerSeptive Biosystems, USA) equilibrated with $10 \mathrm{mM}$ diaminopropane, $\mathrm{pH}$ 9.2. A buffer gradient to $1 \mathrm{M} \mathrm{NaCl-}$ $100 \mathrm{~mm}$ Tris-HCl, $\mathrm{pH} 8.0$, was used for elution.
Fractions containing the protein were further purified by hydrophobic interaction with Butyl-Sepharose (Amersham Pharmacia) by loading at $1.2 \mathrm{M} \mathrm{Na}_{2} \mathrm{SO}_{4}$, followed by elution with a gradient to water. Protein purity was confirmed by SDS-PAGE. The concentration of rOspC was measured with the high-performance gel-permeation chromatography method in a Fractogel EMD column (Merck, Darmstadt, Germany) that monitors UV absorption at $220 \mathrm{~nm}$ [24], with bovine serum albumin (BSA) as reference. This method was used because of the shortage of aromatic amino acids in OspC.

\section{Biotinylation of rOspC}

The purified rOspC proteins from the three strains were biotinylated with ImmunoPure-NHS-Long chain biotin (Pierce, USA), which, dissolved in NN-dimethylformamide, was added to the purified $\mathrm{rOspC}$ protein and to $\mathrm{Na}_{2} \mathrm{CO}_{3}$, and rotated overnight at room temperature. The final concentrations of OspC, biotin and $\mathrm{Na}_{2} \mathrm{CO}_{3}$ were $0.6 \mathrm{mg} / \mathrm{ml}, 0.15 \mathrm{mg} / \mathrm{ml}$ and $0.02 \mathrm{M}$, respectively. Further purification and removal of free biotin was done with gel-permeation chromatography on a Superose 12 column (Amersham Pharmacia) in $0.1 \mathrm{M}$ $\mathrm{Na}_{2} \mathrm{SO}_{4}$.

\section{ELISA}

For rOspC, ELISA microtitration plate wells were coated with recombinant streptavidin (Boehringer Mannheim, Mannheim, Germany) $0.5 \mu \mathrm{g} / \mathrm{ml}$ in PBS overnight at $4^{\circ} \mathrm{C}$. With intervening washes, the following reagents were sequentially incubated on the plates: biotinylated $\mathrm{rOspC}(30 \mathrm{ng} /$ well $)$ in BSA $(5 \mathrm{mg} / \mathrm{ml})$ in $155 \mathrm{mM} \mathrm{NaCl}$-Tween $20 \quad 0.04 \%$ buffer (BSA-NaClTween) for $2 \mathrm{~h}$ at room temperature, serum samples at 1 in 200 dilution in BSA-NaCl-Tween or in $0.1 \mathrm{M}$ NaSCN-BSA-NaCl-Tween overnight at $4^{\circ} \mathrm{C}$, alkaline phosphatase-conjugated rabbit anti-human IgM or IgG (Jackson Immuno Research Laboratories, USA) at 1 in 5000 dilution in BSA-NaCl-Tween for $2 \mathrm{~h}$ and 4-nitrophenylphosphate (Boehringer Mannheim $\mathrm{GmbH}$ ) substrate in $0.1 \mathrm{M}$ diethanolamine- $1 \mathrm{mM} \mathrm{MgCl}_{2}$ buffer, $\mathrm{pH} 10.0$, for $30 \mathrm{~min}$. The absorbance at $405 \mathrm{~nm}$ was measured with a Multiscan photometer (Thermo Labsystems, Helsinki, Finland).

IgM and IgG antibodies against borrelial flagella from B. afzelii DK1 were measured with a commercial flagella-based ELISA kit (Dako, Glostrup, Denmark) modified by using end-point titration of the antibodies, as described earlier [25]. Briefly, sera were diluted serially in three-fold steps for the test and applied to the plates for overnight incubation. The bound antibodies were detected with biotin-labelled goat antihuman IgM or IgG and alkaline phosphatase-labelled streptavidin (Zymed, USA). An end-point titre was obtained at an OD level determined by a cut-off control provided with the kit. 


\section{Patients}

Human serum samples were collected from patients with typical LB, i.e., primary erythema migrans (EM), neuroborreliosis (NB) or Lyme arthritis (LA). In all the patients, the diagnosis of LB was based on the clinical guidelines for diagnosis set out by the Centers for Disease Control and Prevention (CDC; Atlanta, GA, USA) [26]. The clinical diagnoses of NB and LA were confirmed by demonstrating antibodies in the sera by ELISA against WCL from $B$. afzelii strain SK1 (inhouse preparation) and flagella, and, in patients with $\mathrm{NB}$, by demonstrating antibodies to flagella in the cerebrospinal fluid [27]. In all patients with EM, the diagnosis was confirmed by culturing $B$. burgdorferi (13 B. afzelii and 4 B. garinii) from a skin biopsy in BSK-H medium with rifampicin $50 \mu \mathrm{g} / \mathrm{ml}$, phosphomycin $20 \mu \mathrm{g} / \mathrm{ml}$ and amphotericin B $2.5 \mu \mathrm{g} / \mathrm{ml}$ (Sigma). Controls were serum samples from patients with syphilis, Epstein-Barr virus (EBV) infection, rheumatoid factor (RF) positivity, antistreptolysin O (ASO) positivity, high Salmonella or Yersinia enterocolitica antibody titres with positive stool culture, clinically and serologically verified systemic lupus erythematosus (SLE), and samples from healthy blood donors.

\section{Nucleotide sequence accession numbers}

ospC sequences from the B. burgdorferi sensu stricto strain ia, B. afzelii A91 and B. garinii 40 have been assigned to the GenBank database with accession numbers AF501316, AF501318 and AF501317, respectively. Accession numbers of published $o s p C$ sequences used in comparison were X62162 (PKo), X81523 (PLj7), X69594 (PBr), X69593 (TN), X69595 (PBi), X83555 (B. pacificus), X69596 (B31), X81524 (T255), L42893 (297), X81522 (PBre), X80255 (Ple), X83552 (Plud), X83556 (N34), X69592 (T25), X81526 (WABSou), L42892 (ACA1), L42869 (W), X84777 (DK9) and X83553 (Phei).

\section{Statistical analysis}

The Microsoft Excel 2000 program (Microsoft, USA) and Statview 4.5 program (Abacus Concepts, USA) were used for calculations of standard statistics. The coefficient of variation (CV) percentage was determined by dividing the $\mathrm{SD}$ of the $\mathrm{OD}$ values by the mean.

\section{Results}

\section{Nucleotide and protein sequence analysis of OspC}

The identity of the $o s p C$ nucleotide sequences between the Finnish strains ia, A91 and 40 was $76-81 \%$. The deduced amino acid sequences were $67-73 \%$ identical (Fig. 1). When the three amino acid sequences were compared with each other, four amino acid deletions were observed in the OspC sequences of both $B$. burgdorferi ia and B. garinii 40, and three in B. afzelii A91. These deletions were distributed in different parts of the molecules, but so that three of them in strain ia, three in strain A91 and one in strain 40 were in the hypervariable regions of OspC [18]. The C-terminal peptide in $\mathrm{OspC}_{\mathrm{Bg} 40}$ differed from the immunogenic $\mathrm{C}$ terminal decapeptide PVVAESPKKP described by Mathiesen et al. [13] by one amino acid: serine was replaced with threonine. In $\mathrm{OspC}_{\mathrm{Bbia}}$ and $\mathrm{OspC}_{\mathrm{BaA} 91}$, this region was identical with the decapeptide above. In all, $30-33 \%$ of the deduced amino acids were hydrophobic, although the number of aromatic amino acids, which are usually hydrophobic, was low. $\mathrm{OspC}_{\mathrm{Bbia}}$ and $\mathrm{OspC}_{\mathrm{Bg} 40}$ contained two phenylalanines, and $\mathrm{OspC}_{\mathrm{BaA} 91}$ contained three phenylalanines. Only $\mathrm{OspC}_{\mathrm{Bbia}}$ had one tyrosine, and tryptophane was not present in any of the proteins. One cysteine was present in all three proteins, and there were three, two and four histidines in $\mathrm{OspC}_{\mathrm{Bbia}}$, OspC $\mathrm{C}_{\mathrm{BaA} 91}$ and $\mathrm{OspC}_{\mathrm{Bg} 40}$, respectively. The calculated iso-electric points of the mature non-acylated forms of $\mathrm{OspC}_{\mathrm{Bbia}}, \mathrm{OspC}_{\mathrm{BaA} 91}$ and $\mathrm{OspC}_{\mathrm{Bg} 40}$ were $7.96,8.33$ and 7.16 , respectively.
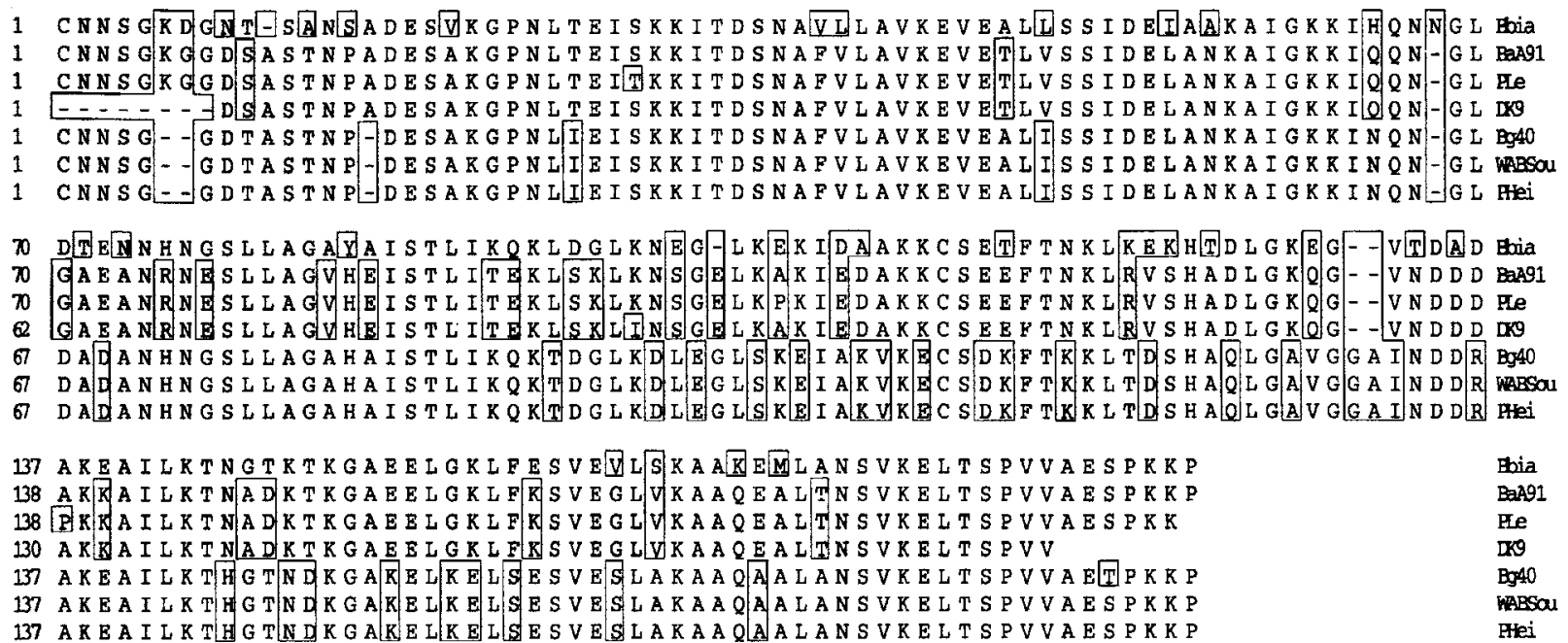

Fig. 1. Comparison of OspC sequences with close homology: the mature proteins of $B$. burgdorferi sensu stricto strain ia (Bbia), $B$. afzelii $\mathrm{A} 91$ (BaA91) and B. garinii 40 (Bg40), and B. afzelii strains Ple and DK9, and B. garinii strains WABSou and PHei. Boxes indicate amino acid heterogeneity. 
The sequences encoding the mature proteins of the three OspCs were compared with the OspC sequences of the 16 OspC serotypes established by Wilske et al. [20], except for strain Pstm, which was not found in the GenBank database. Among these strains, OspC of B. afzelii PLe showed $98.4 \%$ identity with $\mathrm{OspC}_{\mathrm{BaA} 91}$ and $B$. garinii WABSou showed $99.5 \%$ identity with $\mathrm{OspC}_{\mathrm{Bg} 40}$ at the protein level. The OspC sequence of $B$. afzelii ACA1 was identical with $\mathrm{OspC}_{\mathrm{BaA} 91}$, the OspC of $B$. garinii $\mathrm{W}$ was identical with $\mathrm{OspC}_{\mathrm{Bg} 40}$, and the OspC of B. burgdorferi sensu stricto B31 was identical with $\mathrm{OspC}_{\mathrm{Bbia}}$. OspC sequences with close homology are shown in Fig. 1. The published sequence of the OspC of B. afzelii PLe in the GenBank database lacks the last amino acid and B. garinii DK9 lacks the last seven and the first eight amino acids.

\section{IgM ELISA}

Agents known to dissociate inter-protein interactions were tested to improve the binding specificity of $\operatorname{IgM}$ class antibodies in the rOspC ELISA. The ability to differentiate LB from other diseases was monitored in experiments with urea, guanidine or thiocyanate as ingredients in the sample application buffer (data not shown). In preliminary experiments with a small number of sera, the last agent, chosen in the light of a report by McCloskey et al. [28] in which thiocyanate was shown to inhibit binding of antibodies, was promising. Several concentrations of NaSCN (0.1$2 \mathrm{M})$ were tested. The best discrimination between LB patient sera and EBV samples (false positives) was observed with $0.1 \mathrm{M} \mathrm{NaSCN}$ (data not shown). No such effect was observed with $\mathrm{NaSCN}$ when flagella was used as the antigen (data not shown). Depending on the rOspC used, the change in the OD values with or without $\mathrm{NaSCN}$ varied; however, with all the rOspCs, the effect was significantly greater with EBV than with LB patient samples $(\mathrm{p}<0.001)$. With other control samples (Fig. 2), the change in OD resembled that in the LB patient samples. NaSCN decreased the number of false-positive EBV samples from 11 to 7 of 17 . Even more importantly, all the values for the EBV samples remaining above the cut-off value were low positives only (Fig. 2). Increasing the $\mathrm{NaCl}$ concentration instead of adding $\mathrm{NaSCN}$ to the serum dilution buffer did not significantly decrease the OD values of the EBV samples (data not shown).

Subsequently, every sample from patients and controls was examined three times with BSA or $0.1 \mathrm{M} \mathrm{NaSCN}$ BSA buffer in rOspC IgM ELISA. Three rOspC proteins were used separately as antigens. Samples of 19 healthy blood donors were used to define the cut-off value (mean $+2 \mathrm{SD})$. Results were expressed as $\mathrm{OD} /$ cut-off values, which made it possible to combine the results of the three experiments. According to this calculation, every OD/cut-off value $>1$ signifies a positive result. With NaSCN-BSA buffer in ELISA, reactions with one or more of the rOspCs were obtained from $30 \%, 35 \%, 53 \%$ or $53 \%$ of samples from patients with EM at the acute phase, EM at the convalescent phase, NB, or LA, respectively (Table 2). In the same IgM ELISA, $40 \%, 46 \%, 20 \%, 0 \%$ or $5 \%$ of samples from patients with syphilis, EBV, RF positivity, SLE, or of the healthy blood donors, respectively, were positive (Table 2). Positive reactions were mainly observed against $\mathrm{rOspC}_{\mathrm{BaA} 91}$ and $\mathrm{rOspC}_{\mathrm{Bg} 40}$, whereas $\mathrm{rOspC}_{\mathrm{Bbia}}$ detected antibodies infrequently. The positive reactions in the samples from control patients were mostly marginal (Fig. 2). The results from the same serum samples in IgM ELISA with flagella antigen are shown in Table 2.

\section{Reproducibility of rOspC IgM ELISA experiments}

The reproducibility of the test was evaluated by determining the CV of the OD values in three separate ELISA experiments. The total CV was $10.3 \%$ when BSA was used and $7.9 \%$ when NaSCN was present. Thus, NaSCN seemed not only to decrease the nonspecific reactions, but also to improve the CV. No clear difference was seen in the CVs of the various patient groups.

\section{$\operatorname{Ig} G$ ELISA}

The same serum samples that were used in IgM ELISA were tested in IgG ELISA. Control serum samples from patients with high salmonella or yersinia antibody titres and positive stool cultures, and ASO-positive serum samples were also analysed. Of the 17 samples from patients at the acute phase of EM, five reacted

Table 2. Positive IgM ELISA results with rOspC or flagella antigen

\begin{tabular}{|c|c|c|c|c|c|c|c|c|c|c|}
\hline \multirow[b]{2}{*}{ Antigen } & \multirow[b]{2}{*}{$\begin{array}{c}\mathrm{NaSCN} \\
\text { buffer }\end{array}$} & \multicolumn{9}{|c|}{ Number of patients with positive results } \\
\hline & & $\begin{array}{c}\text { EM1 } \\
(\mathrm{n}=17)\end{array}$ & $\begin{array}{c}\text { EM2 } \\
(\mathrm{n}=17)\end{array}$ & $\begin{array}{c}\text { NB } \\
(n=15)\end{array}$ & $\begin{array}{c}\text { LA } \\
(\mathrm{n}=15)\end{array}$ & $\begin{array}{c}\text { SY } \\
(n=10)\end{array}$ & $\begin{array}{c}\text { EBV } \\
(\mathrm{n}=15)\end{array}$ & $\begin{array}{c}\mathrm{RF}+ \\
(\mathrm{n}=10)\end{array}$ & $\begin{array}{c}\text { SLE } \\
(\mathrm{n}=10)\end{array}$ & $\begin{array}{c}\text { BD } \\
(n=19)\end{array}$ \\
\hline rOspC & - & 5 & 7 & 9 & 8 & 4 & 11 & 2 & 0 & 1 \\
\hline rOspC & + & 5 & 6 & 8 & 8 & 4 & 7 & 2 & 0 & 1 \\
\hline Flagella & - & 4 & 3 & 7 & 5 & 1 & 9 & 0 & 0 & 0 \\
\hline
\end{tabular}

rOspC refers to the total number of patients in each group with antibodies to one or more of the rOspC proteins with or without NaSCN in sample buffer. Serum samples were from patients with erythema migrans at the acute phase (EM1), erythema migrans at the convalescent phase (EM2), neuroborreliosis (NB), Lyme arthritis (LA), syphilis (SY), EBV infection (EBV), rheumatoid factor positivity (RF+) or systemic lupus erythematosus (SLE), and from blood donors (BD). 
a

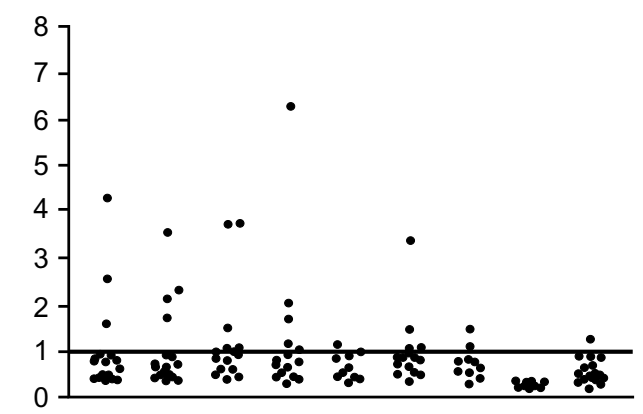

C

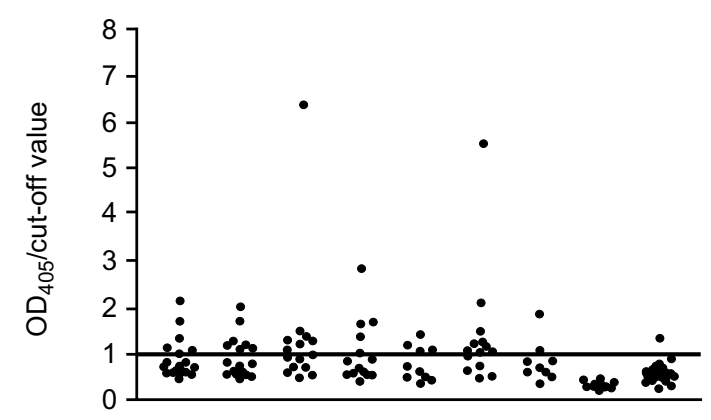

e

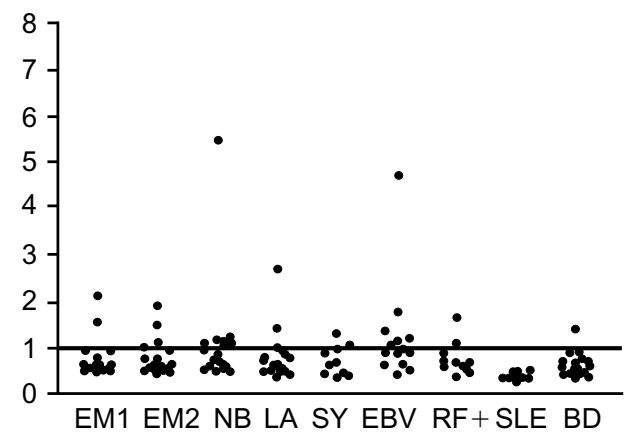

b

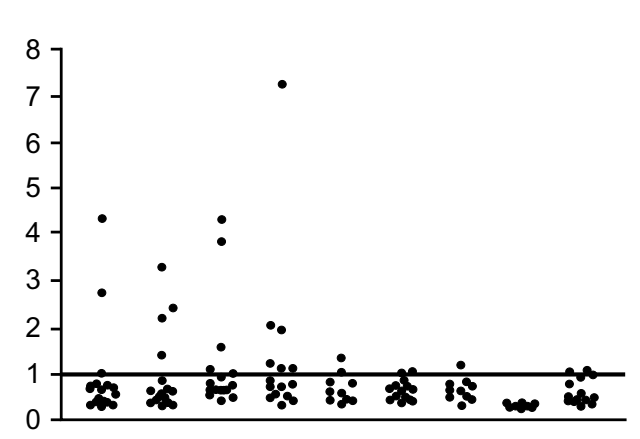

d

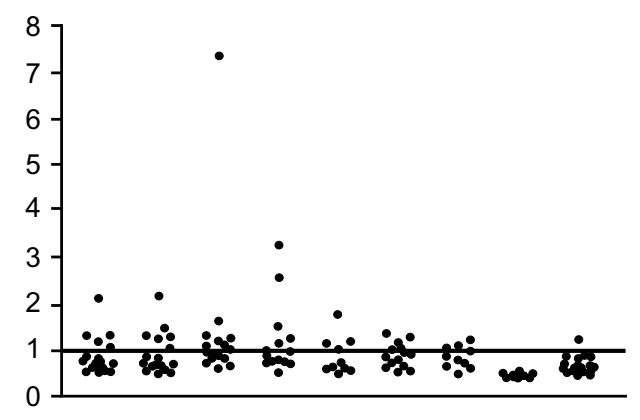

f

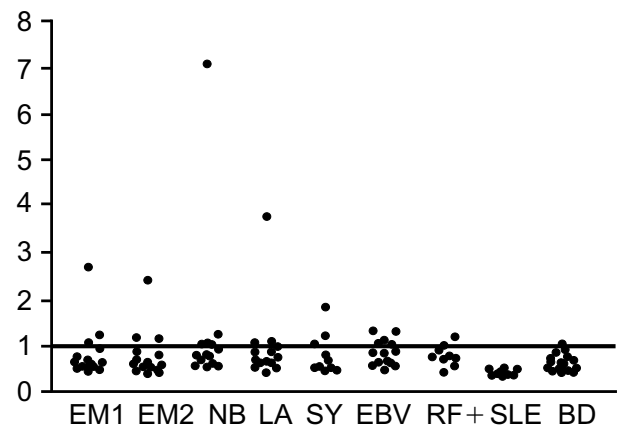

Fig. 2. IgM ELISA results with rOspC proteins from B. afzelii (A91) (a and b), B. garinii (40) (c and d) and B. burgdorferi sensu stricto (ia) (e and f), as antigens. The $\mathrm{OD} /$ cut-off values are shown without (NaSCN-) and with (NaSCN+) sodium thiocyanate. The OD/cut-off values from three distinct ELISA experiments for each patient and control sample are averaged. Serum samples were from patients with erythema migrans at the acute phase (EM1), erythema migrans at the convalescent phase (EM2), neuroborreliosis (NB), Lyme arthritis (LA), syphilis (SY), Epstein-Barr virus infection (EBV), rheumatoid factor positivity (RF+) or systemic lupus erythematosus (SLE), and from healthy blood donors (BD). The level of positivity for OD/cut-off values ( $>1$ ) is indicated by a horizontal line.

with one or more of the rOspCs $(29 \%), 6(35 \%)$ of the 17 EM patients at the convalescent phase, $5(33 \%)$ of 15 with NB, $9(60 \%)$ of 15 with LA, 1 of 10 with syphilis, 0 of 10 with salmonella-positive samples, 1 of 10 with yersinia antibodies, 2 of 10 patients with ASO positivity, 1 of 10 with RF positivity, 3 of the 10 SLE patients and 1 of the 19 healthy blood donors. Of the rOspC proteins, rOspC $\mathrm{BaA}_{\mathrm{Ba}}$ most frequently detected anti-OspC antibodies (Fig. 3).

The same serum samples were tested with flagella IgG ELISA. Four $(23 \%)$ of the 17 samples from patients at both the acute and convalescent phase of EM, 14 $(93 \%)$ of the $15 \mathrm{NB}$ patient samples, $15(100 \%)$ with LA, 1 of 10 with syphilis, 1 of 10 with salmonella infection, 0 of 10 with yersinia infection, 1 of 10 with
ASO positivity, 0 of 10 with RF positivity, 0 of 10 with SLE and 1 of 19 blood donor samples were positive.

\section{Anti-OspC antibodies during the post-treatment period}

In $17 \mathrm{LB}$ patients differences in the antibody responses to $\mathrm{OspC}$ and flagella were evaluated with paired sera at the acute (at diagnosis) and convalescent phases. The convalescence samples were taken 1-6 months after treatment. This group included 3 patients with EM, 10 with NB and 4 with LA. All the patients had LB diagnoses based on the CDC guidelines [26], 15 of the 17 patients had high anti-flagella IgM antibodies at the acute phase, and all 17 patients had high anti-flagella IgG antibodies at the convalescent phase (Table 3). In 
a

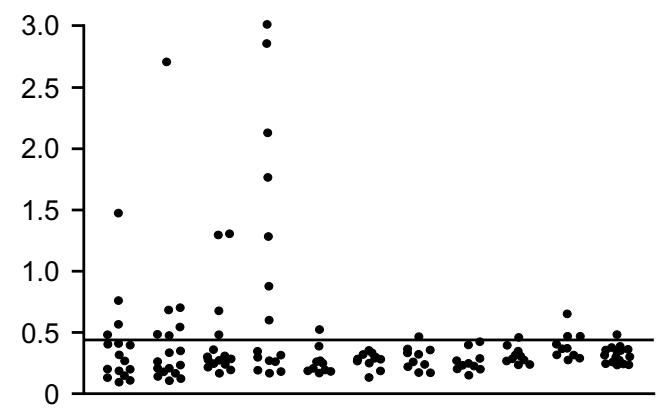

b

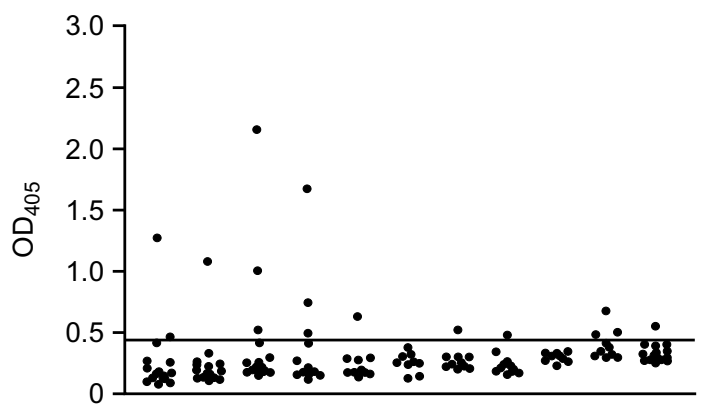

C

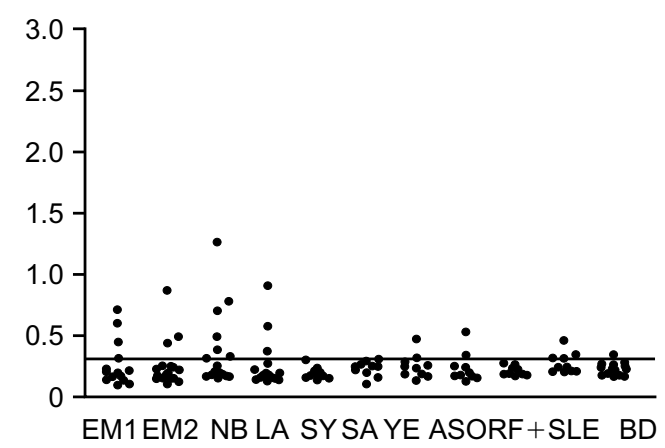

Fig. 3. IgG ELISA results with rOspC proteins from $B$. afzelii (A91) (a), B. garinii (40) (b) and B. burgdorferi sensu stricto (ia) (c), as antigens. $\mathrm{OD}_{405}$ values for each patient and control samples are shown separately. Serum samples were from patients with erythema migrans at the acute phase (EM1), erythema migrans at the convalescent phase (EM2), neuroborreliosis (NB), Lyme arthritis (LA), syphilis (SY), salmonella (SA) or yersinia (YE) infection, antistreptolysin O positivity (ASO), rheumatoid factor positivity $(\mathrm{RF}+)$ or systemic lupus erythematosus (SLE), and from healthy blood donors (BD). The cut-off level (mean +2 SD of BD samples) for each antigen is indicated by a horizontal line.

IgM ELISA with NaSCN-BSA buffer, 12 (71\%) of the 17 patients were positive with one or more of the rOspCs at the acute phase. Of the rOspCs, rOspC $\mathrm{BaA}_{\mathrm{Ba}}$ evoked a positive reaction most frequently (Table 3), although in IgM ELISA immunoreactivity toward two or three of the rOspCs was often seen. In the convalescent phase, nine patients had retained and one gained IgM antibody positivity toward flagella. There were two seroreversions and two seroconversions toward rOspCs.
Table 3. Positive results of $\operatorname{IgM}$ and IgG ELISA with rOspC from B. afzelii (A91), B. garinii (40) and $B$. burgdorferi sensu stricto (ia) and flagella antigen

\begin{tabular}{|c|c|c|c|c|}
\hline \multirow[b]{2}{*}{ Antigen } & \multicolumn{4}{|c|}{ Number of patients with positive results } \\
\hline & $\begin{array}{c}\text { IgM } \\
\text { acute } \\
(n=17)\end{array}$ & $\begin{array}{c}\text { IgM } \\
\text { convelescent } \\
(\mathrm{n}=17)\end{array}$ & $\begin{array}{c}\text { IgG } \\
\text { acute } \\
(n=17)\end{array}$ & $\begin{array}{c}\text { IgG } \\
\text { convelescen } \\
(\mathrm{n}=17)\end{array}$ \\
\hline $\mathrm{OspC}_{\mathrm{BaA} 91}$ & $11(5)$ & $11(4)$ & $9(6)$ & $12(7)$ \\
\hline $\mathrm{OspC}_{\mathrm{Bg} 40}$ & $7(1)$ & $8(0)$ & $3(0)$ & $6(1)$ \\
\hline OspC $_{\text {Bbia }}$ & $2(0)$ & $3(0)$ & $1(0)$ & $1(0)$ \\
\hline OspC $_{\text {Total }}$ & 12 & 12 & 9 & 13 \\
\hline Flagella & 15 & 10 & 13 & 17 \\
\hline
\end{tabular}

Serum samples were from patients with LB at the acute and convalescent phases. NaSCN was used in IgM ELISA. OspC $\mathrm{Cotal}_{\text {Tol }}$ refers to the total number of patients in each group with antibodies to any of the rOspC proteins. The number of OD/cut-off values that were positive with one OspC variant only is shown in parentheses.

IgG ELISA was also performed for the same 17 paired serum samples. Nine $(53 \%)$ of the 17 patients had positive reactions with one or more of the rOspCs in the acute phase, and $13(76 \%)$ of 17 in the convalescent phase (Table 3 ). The majority of positive immunoreactions were against $\mathrm{rOspC}_{\mathrm{BaA} 91}, 9$ of 17 in the acute phase and 12 of 17 during the convalescent phase (Table 3). In the convalescent phase, one serum sample was positive for $\mathrm{rOspC}_{\mathrm{Bg} 40}$ only; otherwise, $\mathrm{rOspC}_{\mathrm{BaA} 91}$ would have covered the antibody responses in $\mathrm{rOspC}$ IgG ELISA.

\section{Discussion}

This study showed that in serological assays, the abilities of rOspC and flagella to detect IgM antibodies during early LB were comparable. With both antigens, IgM serology is complicated by false-positive reactions, a frequent problem with IgM antibodies in general. The results of the present study suggest that $\mathrm{NaSCN}$ may be able to decrease non-specific immunoreactivity and, thus, to improve the IgM serodiagnosis. In contrast, the sensitivity of OspC IgG serology remained lower than that of the conventional flagella antigen, even though the various OspCs covered all three pathogenic species in the antigen panel. Most probably, the results reflect the high sequence heterogeneity of OspC.

In the early stages of LB, antibodies against borrelial proteins are observed in only a small proportion of patients [3]. In the present study, OspC ELISA was compared with flagella ELISA, because the performance of this method has been at the same level as that of other commercially available ELISA methods [29]. In the EM serum samples, sensitivity and specificity of rOspC seemed to be approximately equal to those of the flagella antigen. In previous studies, IgM antibodies to rOspC have been observed in $25-80 \%$ of patients with EM [4-9, 14, 30] and in $48-72 \%$ of patients with NB [4,6]. The variability of these results may be explained partly by the various stages of the disease represented by the patient samples or by the origin 
(subspecies) of OspC used as antigen, or both. In the present study, IgM antibodies were most frequently detected with rOspC from B. afzelii and B. garinii, but only rarely with rOspC from $B$. burgdorferi sensu stricto. Recently, other recombinant proteins have also been studied as antigens in IgM serology. An earlier study on recombinant FlaA proteins [31] showed 20$27 \%$ or $58 \%$ sensitivity in IgM ELISA for EM or NB samples, respectively. Rauer et al. [8] combined recombinant OspC and recombinant 14-kDa flagellin fragment as ELISA antigens and observed an additive effect on the sensitivity of IgM serology.

In the present study, the specificity of OspC ELISA was evaluated with control samples from patients with syphilis, EBV infection, RF positivity or SLE, and from healthy blood donors. EBV was especially problematic, as the majority of samples from patients seemed to cause non-specific reactions. Other controls produced mainly low false-positive results. The proportion of positive EBV patients in rOspC ELISA decreased from $73 \%$ to $46 \%$ when NaSCN-BSA buffer was used, and those still positive were only marginally above the cut-off value. NaSCN did not have a significant effect in the other control samples. Previously, Goossens et al. [29] have suggested that, for serodiagnosis of LB, the exclusion of false positivity by targeted assays for EBV, syphilis infections and RF positivity would be more successful than the two-tier assay by successive ELISA and WB recommended by CDC [32].

A prominent feature of $\operatorname{IgG}$ serology was the predominance of IgG immune reactions against OspC from $B$. afzelii. The high prevalence of $B$. afzelii in Scandinavia may account for this finding [23]. In published studies, the proportion of positive samples against single recombinant OspC has varied between $5 \%$ and $42 \%$ early in the disease $[4-6,9,14]$ and between $6 \%$ and $51 \%$ in disseminated disease $[4,6]$. The findings in the present study concur with these results, showing $6-35 \%$ positive results in the acute phase and $18-53 \%$ in the convalescent phase of LB, depending on the various rOspC antigens employed. It is likely that maturation of the $\operatorname{IgG}$ immune response with time would direct the specificity of the antibodies preferentially toward epitopes of the immunising strain. Fung et al. [4] found an IgG response in rOspC ELISA (rOspC from B. burgdorferi sensu stricto strain 297) in chronic LA patients but less frequently in chronic NB patients. In keeping with these results, in the present rOspC IgG ELISA, the patients with LA had antibodies more frequently than the patients with EM or NB. This may have been due to the more chronic stage of the disease in LA than in EM or NB.

The present study used an ELISA with streptavidincoated plates and rOspC bound to it via a biotin tag. This procedure improved the binding of $\mathrm{rOspC}$ to the ELISA plate surface (data not shown). Alternatively,
Wienecke et al. [9] successfully used covalent coupling of rOspC to plastic. The poor binding of the rOspC constructs to plain plastic in the present study may be associated with the low content of aromatic amino acids in OspC. Similarly, weak binding of OspC to nitrocellulose WCL immunoblot strips in the presence of SDS in the transfer buffer was observed (data not shown).

In conclusion, this study implies that, depending on the epidemiological situation, all pathogenic borrelial species should be covered if OspC antigens are employed in the serology of LB. We suggest that in the European context, on account of the heterogeneity of OspC, a polyvalent antigen with several OspC variants from at least $B$. afzelii and $B$. garinii is needed to improve the serodiagnosis of LB.

We thank Dr Klaus Hedman for providing the EBV serum samples, Dr Matti Viljanen for donating the strains of B. burgdorferi sensu lato used in this study, and Drs Heikki Julkunen and Aaro Miettinen for the SLE samples. The English language was checked by Mrs Jean Margaret Perttunen. This study was supported by grants from the National Technology Agency (Tekes), Helsinki, Finland; Helsinki Central Hospital Research Funds; the Clinical Research Institute of Helsinki University Central Hospital; the Paulo Foundation and Finska Läkaresällskapet, Helsinki, Finland.

\section{References}

1. Sigal LH. Pitfalls in the diagnosis and management of Lyme disease. Arthritis Rheum 1998; 41: 195-204.

2. Brown SL, Hansen SL, Langone JJ. Role of serology in the diagnosis of Lyme disease. JAMA 1999; 282: 62-66.

3. Aguero-Rosenfeld ME, Nowakowski J, Bittker S, Cooper D, Nadelman RB, Wormser GP. Evolution of the serologic response to Borrelia burgdorferi in treated patients with culture-confirmed erythema migrans. J Clin Microbiol 1996; 34: 1-9.

4. Fung BP, McHugh GL, Leong JM, Steere AC. Humoral immune response to outer surface protein $\mathrm{C}$ of Borrelia burgdorferi in Lyme disease: role of the immunoglobulin $\mathrm{M}$ response in the serodiagnosis of early infection. Infect Immun 1994; 62: 3213-3221.

5. Magnarelli LA, Fikrig E, Padula SJ, Anderson JF, Flavell RA. Use of recombinant antigens of Borrelia burgdorferi in serologic tests for diagnosis of Lyme borreliosis. J Clin Microbiol 1996; 34: 237-240.

6. Mathiesen MJ, Christiansen M, Hansen K, Holm A, Åsbrink E, Theisen M. Peptide-based OspC enzyme-linked immunosorbent assay for serodiagnosis of Lyme borreliosis. J Clin Microbiol 1998; 36: 3474-3479.

7. Padula SJ, Dias F, Sampieri A, Craven RB, Ryan RW. Use of recombinant OspC from Borrelia burgdorferi for serodiagnosis of early Lyme disease. J Clin Microbiol 1994; 32: 1733-1738.

8. Rauer S, Spohn N, Rasiah C, Neubert U, Vogt A. Enzymelinked immunosorbent assay using recombinant OspC and the internal 14-kDa flagellin fragment for serodiagnosis of early Lyme disease. J Clin Microbiol 1998; 36: 857-861.

9. Wienecke CA, Lovrich SD, Callister SM, Jobe DA, Marks JA, Schell RF. Evaluation of whole-cell and OspC enzymelinked immunosorbent assays for discrimination of early Lyme Borreliosis from OspA vaccination. J Clin Microbiol 2000; 38: 313-317.

10. Hauser U, Lehnert G, Wilske B. Diagnostic value of proteins of three Borrelia species (Borrelia burgdorferi sensu lato) and implications for development and use of recombinant antigens for serodiagnosis of Lyme Borreliosis in Europe. Clin Diagn Lab Immunol 1998; 5: 456-462.

11. Engstrom SM, Shoop E, Johnson RC. Immunoblot interpretation criteria for serodiagnosis of early Lyme disease. J Clin 
Microbiol 1995; 33: 419-427.

12. Ikushima M, Kawahashi S, Ohzeki $\mathrm{Y}$ et al. A new specific serodiagnosis system for Lyme disease: use of synthetic peptides derived from outer surface protein $\mathrm{C}$ of Borrelia burgdorferi. Opportunistic Pathology 1997; 9: 21-25.

13. Mathiesen MJ, Holm A, Christiansen M et al. The dominant epitope of Borrelia garinii outer surface protein $\mathrm{C}$ recognized by sera from patients with neuroborreliosis has a surfaceexposed conserved structural motif. Infect Immun 1998; 66: 4073-4079.

14. Magnarelli LA, Ijdo JW, Padula SJ, Flavell RA, Fikrig E. Serologic diagnosis of Lyme borreliosis by using enzymelinked immunosorbent assays with recombinant antigens. J Clin Microbiol 2000; 38: 1735-1739.

15. Gomes-Solecki MJC, Dunn JJ, Luft BJ et al. Recombinant chimeric Borrelia proteins for diagnosis of Lyme disease. $J$ Clin Microbiol 2000; 38: 2530-2535.

16. Gomes-Solecki MJC, Wormser GP, Persing DH et al. A firsttier rapid assay for the serodiagnosis of Borrelia burgdorferi infection. Arch Intern Med 2001; 161: 2015-2020.

17. Jauris-Heipke S, Liegl G, Preac-Mursic V et al. Molecular analysis of genes encoding outer surface protein C (OspC) of Borrelia burgdorferi sensu lato: relationship to ospA genotype and evidence of lateral gene exchange of $\operatorname{spc} C \mathrm{~J}$ Clin Microbiol 1995; 33: 1860-1866.

18. Livey I, Gibbs CP, Schuster R, Dorner F. Evidence for lateral transfer and recombination in OspC variation in Lyme disease Borrelia. Mol Microbiol 1995; 18: 257-269.

19. Theisen M, Frederiksen B, Lebech A-M, Vuust J, Hansen K. Polymorphism in ospC gene of Borrelia burgdorferi and immunoreactivity of OspC protein: implications for taxonomy and for use of OspC protein as a diagnostic antigen. $J$ Clin Microbiol 1993; 31: 2570-2576.

20. Wilske B, Busch U, Fingerle $\mathrm{V}$ et al. Immunological and molecular variability of OspA and OspC. Implications for Borrelia vaccine development. Infection 1996; 24: 208-212.

21. Wilske B, Jauris-Heipke S, Lobentanzer R et al. Phenotypic analysis of outer surface protein C (OspC) of Borrelia burgdorferi sensu lato by monoclonal antibodies: relationship to genospecies and OspA serotype. J Clin Microbiol 1995; 33: $103-109$
22. Wilske B, Preac-Mursic V, Göbel UB et al. An OspA serotyping system for Borrelia burgdorferi based on reactivity with monoclonal antibodies and OspA sequence analysis $J$ Clin Microbiol 1993; 31: 340-350.

23. Junttila J, Peltomaa M, Soini H, Marjamäki M, Viljanen MK Prevalence of Borrelia burgdorferi in Ixodes ricinus ticks in urban recreational areas of Helsinki. J Clin Microbiol 1999 37: $1361-1365$.

24. Hayakawa $\mathrm{K}$, Masuko $\mathrm{M}$, Mineta $\mathrm{M}$ et al. Serum protein determination by high-performance gel-permeation chromatography. J Chromatr B Biomed Sci Appl 1997; 696: 19-23.

25. Seppälä IJT, Kroneld R, Schauman K, Forsen K-O, Lassenius R. Diagnosis of Lyme borreliosis: non-specific serological reactions with Borrelia burgdorferi sonicate antigen caused by IgG2 antibodies. J Med Microbiol 1994; 40: 293-302.

26. Wharton M, Chorba TL, Vogt RL, Morse DL, Buehler JW Case definitions for public health surveillance. $M M W R$ Morbid Mortal Wkly Rep 1990; 39: 19-21.

27. Peltomaa M, Pyykkö I, Seppälä I, Viljanen L, Viljanen M Lyme borreliosis, an etiologic factor of sensorineural hearing loss? Eur Arch Otorhinolaryngol 2000; 257: 317-322.

28. McCloskey N, Turner MW, Goldblatt TD. Correlation between the avidity of mouse-human chimeric IgG subclass monoclonal antibodies measured by solid-phase elution ELISA and biospecific interaction analysis (BIA). J Immunol Methods 1997; 205: 67-72.

29. Goossens HAT, van den Bogaard AE, Nohlmans MKE Evaluation of fifteen commercially available serological tests for diagnosis of Lyme borreliosis. Eur J Clin Microbiol Infect Dis 1999; 18: 551-560.

30. Gerber MA, Shapiro ED, Bell GL, Sampieri A, Padula SJ. Recombinant outer surface protein C ELISA for the diagnosis of early Lyme disease. J Infect Dis 1995; 171: 724-727.

31. Panelius J, Lahdenne P, Saxen H, Heikkilä T, Seppälä I. Recombinant Flagellin A proteins from Borrelia burgdorferi sensu stricto, $B$. afzelii, and B. garinii in the serodiagnosis of Lyme borreliosis. J Clin Microbiol 2001; 39: 4013-4019.

32. Centers for Disease Control and Prevention. Recommendations for test performance and interpretation from the Second National Conference on Serologic Diagnosis of Lyme Disease. MMWR Morbid Mortal Wkly Rep 1995; 44: 590-591. 